\title{
Evaluation de la connaissance et de la mise en ouvre des bonnes pratiques d'hygiène par les populations riveraines du lac Nokoué (Sud-Bénin)
}

\author{
Sèdami Pivot Amour SACHI ${ }^{1 *}$, Innocent BOKOSSA YAOU ${ }^{1}$, Comlan Célestin K. TCHEKESSI ${ }^{1}$, \\ Sèmèvo Bleck Jultesse BANON ${ }^{1}$, Roseline BLEOUSSI ${ }^{1}$, Aurelle Anayce DJOGBE ${ }^{1}$ et \\ Guy Apollinaire MENSAH ${ }^{2}$ \\ ${ }^{l}$ Laboratoire de Microbiologie et des Technologies Alimentaires (LA.MI.T.A) du Département de Biologie Végétale de la \\ Faculté des Sciences et Techniques (FAST) de l'Université d'Abomey-Calavi (UAC); 04BP 1107 Cotonou (Bénin); \\ ${ }^{2}$ Institut National des Recherches Agricoles du Bénin (INRAB) ; 01 BP 884 Recette Principale, Cotonou 01 (Bénin). \\ *Auteur correspondant; E-mail : amoursachi@gmail.com;01BP : 525 Tri postal ; Ville : Cotonou ; Pays : Rép. Bénin,
} Tél. : (+229) 96726714

\section{RESUME}

Au Bénin, les acteurs de la pêche continentale vivent en communautés dans des villages installés aux abords immédiats des cours d'eau ou dans des cases construites sur pilotis en pleine eau. Tel est le cas des villages lacustres du lac Nokoué. Ces populations affectent directement ou indirectement la qualité de ces cours d'eau de par leurs rejets et aussi de par leurs différentes activités. L'objectif de l'étude a été d'apprécier la connaissance et la mise en œuvre des bonnes pratiques d'hygiène et des bonnes pratiques de gestion environnementale par les populations du lac Nokoué. Pour atteindre cet objectif, une fiche d'enquête a été adressée aux dites populations. Un total de 280 personnes a été enquêté. Les données collectées ont été analysées grâce au logiciel SPSS 16 et au tableur Excel. Les résultats obtenus montrent que plus de la moitié des enquêtés $(56,79 \%)$ n'ont jamais suivi une formation sur les règles d'hygiène. 84,29\% des enquêtés n'ont pas accès aux latrines et font leurs besoins directement dans les eaux. $82,5 \%$ et $68,93 \%$ des enquêtés y rejettent respectivement leurs déchets liquides et solides. La mise en œuvre rigoureuse des bonnes pratiques d'hygiène n'est donc pas observée par la plupart des enquêtés.

(C) 2016 International Formulae Group. All rights reserved.

Mots clés : Bonnes pratiques d’hygiène, enquête, populations lacustres, lac Nokoué, Bénin.

\section{Evaluation of knowledge and the implementation of the good practices of hygiene by the bordering populations of the lake Nokoué (South-Benin)}

\begin{abstract}
In Benin, continental fishing actors live in communities in villages settled along water sources or huts built on stilts in water. This is the case of the lakeside villages of Nokoué Lake. These populations impact directly or indirectly the water sources quality through their discharges and different activities as well. The objective of this study was to access knowledge on the implementation of good hygiene practices as well as good environmental management practices of the populations on the Nokoué Lake. 280 people were
\end{abstract}


interviewed and data collected, analyzed thanks to SPSS 16 software and Excel table. The results showed that more than half interviewees (56.79\%) never attended training on hygiene rules. 84.29\% of the interviewees not have access to latrines and ease themselves directly in waters. $82.5 \%$ and $68.93 \%$ of the interviewees release in the waters their liquid and solid thrash respectively. Therefore the strict implementation of the good hygiene practices is not respected by most of the interviewees.

(C) 2016 International Formulae Group. All rights reserved.

Keywords: Good hygiene practices, interview, lake community, Nokoué Lake, Benin.

\section{INTRODUCTION}

En Afrique de l'Ouest et au Sud du Sahara, se trouve une séquence continue de lacs et lagunes dont les ressources halieutiques sont très variées et intensément utilisées par les populations riveraines (Pombo et al., 2002; Gnohossou, 2006). Le réseau hydrographique du Sud-Bénin est composé de fleuves, de lacs et lagunes qui constituent un écosystème complexe et dynamique avec une productivité biologique très variée et élevée (Akambi, 1990 cité par Gnohossou, 2006). Il dispose d'une façade maritime d'environ 120 $\mathrm{km}$ et d'un réseau lagunaire côtier subséquent : la lagune de Porto-Novo, le lac Nokoué, la lagune de Cotonou et le lac Ahémé. Au Bénin, les pêcheries continentales contribuent pour environ 75 à $80 \%$ à la production halieutique nationale (Gnohossou, 2006 ; Rurangwa et al., 2014) et le lac Nokoué est le cours d'eau le plus productif du Bénin (Lalèyè et Moreau, 2004). La pêche continentale constitue également une source génératrice de revenus pour les populations riveraines dont la principale activité est la pêche (Egounlety, 2005).

En effet, les pêcheurs et autres acteurs de la filière pêche vivent en communauté dans les villages installés aux abords immédiats des plans d'eau ou dans des cases construites sur pilotis en pleine eau. Leurs rejets (excréments et urine) et déchets domestiques (solides et liquides) sont déversés directement ou indirectement dans l'eau. Ce qui constitue une source de pollution (contaminations chimique et microbiologique) de ces eaux (Sèdogbo, 2004 ; Agonkpahoun, 2006 ; Dovonou et al., 2011; Mègnon et al., 2012; Adjahouinou et Fiogbé 2014). Au regard de toutes ces observations, il s'avère donc nécessaire de contrôler l'action desdites populations sur la qualité hygiénique de ces cours d'eau.

L'objectif de l'étude est d'évaluer à travers une enquête de terrain, la connaissance et la maîtrise des règles d'hygiène par les populations vivant aux abords du lac Nokoué et leur influence sur la qualité de ce dernier.

\section{MATERIEL ET METHODES}

Le cadre géographique de la présente étude est le lac Nokoué (Figure 1). Il est situé au Sud-Est du Bénin et est compris entre les parallèles $6^{\circ} 20^{\prime}$ et $6^{\circ} 30^{\prime}$ Nord et les méridiens $2^{\circ} 20^{\prime}$ et $2^{\circ} 35^{\prime}$ Est. S'étendant sur les départements de l'Ouémé, de l'Atlantique et du Littoral, il est limité au Sud par les villes de Cotonou et de Sèmè-Kpodji et la lagune de Cotonou; au Nord par les communes des Aguégués et de Sô-Ava, le fleuve Ouémé et la rivière Sô ; à l'Est par la lagune de PortoNovo et à l'Ouest par la commune d'AbomeyCalavi. Le chenal de Cotonou et le canal de Totchè relient respectivement le lac à l'Océan Atlantique et à la lagune de Porto-Novo. D'une superficie de $150 \mathrm{~km}^{2}$ à l'étiage, il est long de $20 \mathrm{~km}$ dans sa direction Est-Ouest et de $11 \mathrm{~km}$ dans sa direction Nord-Sud (Mama, 2010 ; Dovonou et al., 2011).

L'enquête s'était déroulée du 6 Avril 2015 au 5 Mai 2015 soit pendant 30 jours. Elle a été conduite dans sept principaux villages situés aux abords du lac Nokoué. Ces villages ont été choisis à l'issue d'une préenquête à cause de leur proximité du lac, de la masse de leur population et de leur intensité de pêche. Il s'agit des villages lacustres de : 
- Ganvié, Sô-Tchanhoué, Sô-Zounko et Houèdo-Aguékon dans la commune de SôAva, département de l'Atlantique.

- Tchonvi et Kétonou dans la commune de Sèmè-Kpodji, département de l'Ouémé.

- Agbato dans la commune de Cotonou, département du Littoral.

\section{Echantillonnage}

L'enquête a été réalisée auprès de 280 personnes (soit une personne par ménage afin d'éviter la répétition des informations au sein d'un même ménage). Cette taille de l'échantillon a été déterminée en utilisant la formule de Dagnelie (1998) $: n=4 p(1-p) /$ $\boldsymbol{d}^{2}$; où $\boldsymbol{n}$ est le nombre total de ménages à enquêter, $\boldsymbol{d}$ la marge d'erreur fixée à 0,05 et $\boldsymbol{p}$, la proportion des ménages vivant dans la zone étudiée au sein de l'ensemble des ménages installés autour du lac Nokoué. Le paramètre $\boldsymbol{p}$ a été déterminé sur la base des résultats du troisième Recensement Général de la Population et de l'Habitat (RGPH3).

Par exemple, la taille totale de ménages installés autour du lac Nokoué était d'environ 14371 (Djènontin et Guidibi, 2006). Parmi eux, 11327 étaient des villages choisis (INSAE, 2004). La répartition de la taille de ménages à enquêter par municipalité était proportionnelle à la taille de ménages vivant autour du lac. Si $\boldsymbol{x}_{i}$ est la taille de ménages d'une municipalité, la proportion $\boldsymbol{q}_{i}$ de ménages de cette municipalité par rapport à la taille totale $N$ de ménages vivant autour du lac a été déterminée par la formule : $\boldsymbol{q}_{i}=\boldsymbol{x}_{i} / \boldsymbol{N}$. Le nombre $\boldsymbol{n}_{\boldsymbol{i}}$ de ménages à enquêter dans cette municipalité était alors $\boldsymbol{n}_{\boldsymbol{i}}=\boldsymbol{n} \times \boldsymbol{q}_{\boldsymbol{i}}$. Le Tableau 1 présente cette répartition.

\section{Technique d'enquête}

Les autorités politico-administratives (chef d'arrondissement, chef de village et délégués) de ces localités ont été sensibilisées au préalable à l'objectif de ladite enquête. Les investigations auprès de ces personnes ont consisté en des interviews libres, à l'administration des questionnaires et aux entretiens en langues nationales.

\section{Traitement des données collectées}

Les données collectées à l'issue de cette enquête ont été analysées grâce au logiciel SPSS 16 et au tableur Excel.

\section{RESULTATS \\ Profil sociodémographique des enquêtés}

Le profil sociodémographique des personnes enquêtées est consigné dans le Tableau 2. La différence entre les personnes enquêtées de sexe féminin et celles de sexe masculin était de 2,86\% (Tableau 2).

Les personnes enquêtées dont l'âge était compris entre 25 et 55 ans étaient près de 2,33 fois supérieures à celles dont l'âge était inférieur à 25 ans et près de 10,83 fois supérieures à celles dont l'âge était supérieur à 55 ans (Tableau 2).

Les personnes enquêtées dont les maisons étaient construites sur le lac étaient plus de 4,71 fois élevées que celles installées sur les berges (Tableau 2).

\section{Accès des enquêtés aux toilettes (latrines)}

L'accès des personnes enquêtées aux latrines est présenté par la Figure 2. Les personnes enquêtées n'ayant pas accès aux latrines pour leurs besoins (selle et urine) étaient plus de 5,36 fois élevées que celles ayant accès à des latrines avec fosses installées sur les berges (Figure 2).

\section{Gestion des déchets domestiques solides et liquides par les enquêtés}

Les Figures 3 et 4 présentent l'accès des personnes enquêtées aux poubelles et la gestion de leurs déchets liquides (eaux usées). Les personnes enquêtées qui ne possédaient pas du tout de poubelles étaient près de 2,22 fois plus élevées que celles qui en possédaient (Figure 3). Ces personnes jetaient leurs ordures n'importe où (dans le lac ou sur les berges). Quant aux personnes enquêtées possédant de poubelles, elles géraient ellesmêmes leurs poubelles en amenant les ordures sur les berges pour les brûler ou pour les laisser. Toutes les personnes enquêtées qui étaient installées sur le lac jetaient leurs eaux 
usées dans le lac tandis que, toutes celles qui étaient installées sur les berges les jetaient sur les berges (Figure 4).

\section{Gestion des déchets résultant de leurs principales activités}

$60,71 \%$ des personnes enquêtées avaient répondu que les anciens branchages utilisés pour faire les acadjas étaient délaissés dans le lac alors que les autres avaient répondu que ces branchages étaient ramenés sur les berges pour être brulés.

$53,93 \%$ des personnes enquêtées pratiquaient l'élevage de certains animaux domestiques (porcins, ovins, volaille, bovins) qui étaient soit dans des enclos installés sur le lac ou sur les berges ou soit laissés à euxmêmes. Les fientes de ces animaux allaient directement dans le lac ou drainés des berges vers le lac par les eaux de ruissellement.

$29,64 \%$ des personnes enquêtées convoyaient des produits pétroliers à travers le lac et avaient souligné que ces produits pouvaient se déverser dans le lac lors du transport.

\section{Formation sur les règles d'hygiène}

Parmi les personnes enquêtées, seuls $43,21 \%$ avaient reçu une formation sur les règles d'hygiène. Leur répartition en fonction de la durée de leur dernière formation est présentée par la Figure 5.

Les personnes enquêtées qui n'avaient pas du tout suivi au moins une fois une formation sur les règles d'hygiène étaient près de 1,32 fois plus élevées que celles qui en avaient reçue (Figure 5). Celles qui avaient reçu une formation récente (datant de six mois au plus) sur les règles d'hygiène étaient respectivement plus de la moitié de celles qui avaient reçu une formation datant de deux ans et plus du tiers de celles qui avaient reçu une formation de plus de deux ans (Figure 5). La différence entre les personnes qui avaient reçu une formation datant de six mois au plus et les personnes qui avaient reçu une formation datant d'un an était de 1,80\% (Figure 5). Les personnes enquêtées qui avaient reçu une formation datant de plus de deux ans étaient près de 2,23 fois supérieures à celles qui avaient reçu une formation datant d'un an et près de 1,49 fois plus élevées que celles qui avaient reçu une formation datant de deux ans (Figure 5). La différence entre les personnes enquêtées qui avaient reçu une formation datant de deux ans et celles qui avaient reçu une formation datant d'un an sur les règles d'hygiène était de 3,93\% (Figure 5).

Tableau 1 : Nombre de ménages enquêtés au sein de chaque village de la zone d'étude.

\begin{tabular}{ccc}
\hline Localités ou villages lacustres & $\begin{array}{c}\text { Nombre de ménages } \\
\text { (INSAE, 2004) }\end{array}$ & Nombre d'enquêtées \\
\hline Ganvié & 3521 & 87 \\
Sô-Zounko & 413 & 10 \\
Sô-Tchanhoué & 2318 & 57 \\
Houèdo-Aguékon & 2010 & 50 \\
Agbato & 1336 & 33 \\
Kétonou & 964 & 24 \\
Tchonvi & 765 & 19 \\
\hline
\end{tabular}


Tableau 2 : Profil sociodémographique des enquêtés.

\begin{tabular}{|c|c|c|}
\hline \multicolumn{2}{|c|}{ Caractéristiques } & \multirow{2}{*}{$\frac{\text { \% des enquêtés }}{48,57 \%}$} \\
\hline & Masculin & \\
\hline \multirow[t]{2}{*}{ Genre ou sexe } & Féminin & $51,43 \%$ \\
\hline & $<25$ & $28,21 \%$ \\
\hline \multirow[t]{2}{*}{ Age (ans) } & $25-55$ & $65,72 \%$ \\
\hline & $>55$ & $6,07 \%$ \\
\hline \multirow[t]{2}{*}{ Situation de l'habitat } & Sur le lac & $82,5 \%$ \\
\hline & Sur les berges & $17,5 \%$ \\
\hline
\end{tabular}

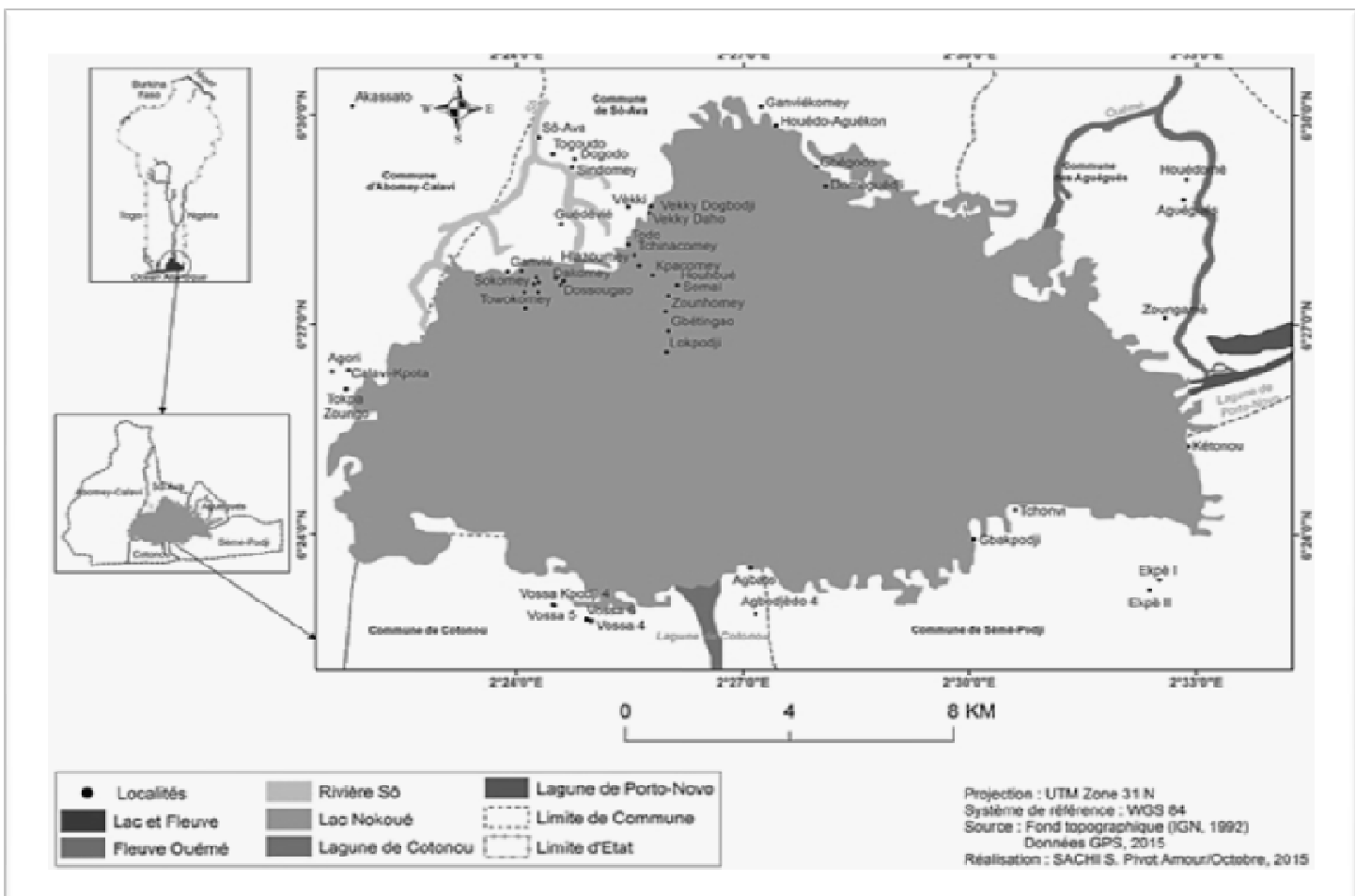

Figure 1 : Carte du lac Nokoué (Sachi, 2015).

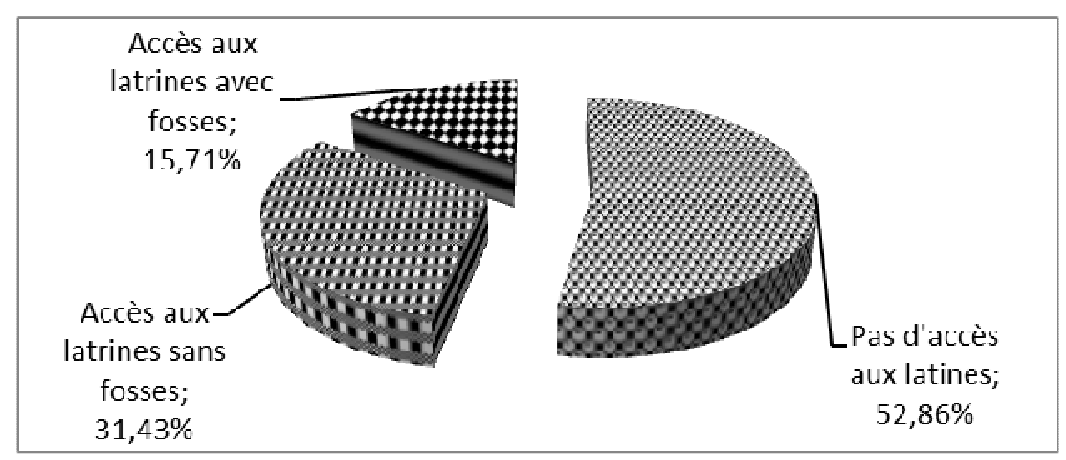

Figure 2 : Répartition des personnes enquêtées ayant accès ou non aux latrines. 


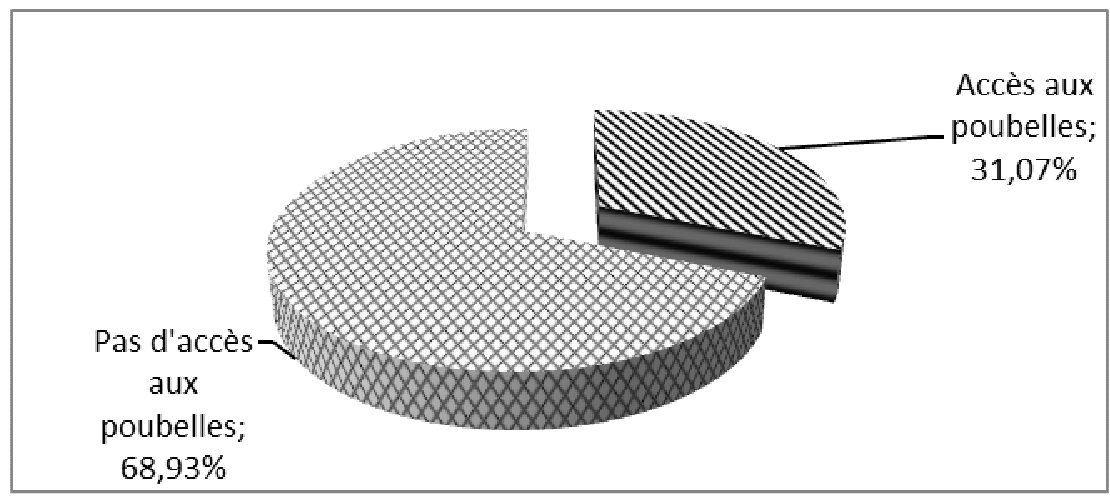

Figure 3 : Répartition des personnes enquêtées ayant accès ou non aux poubelles.

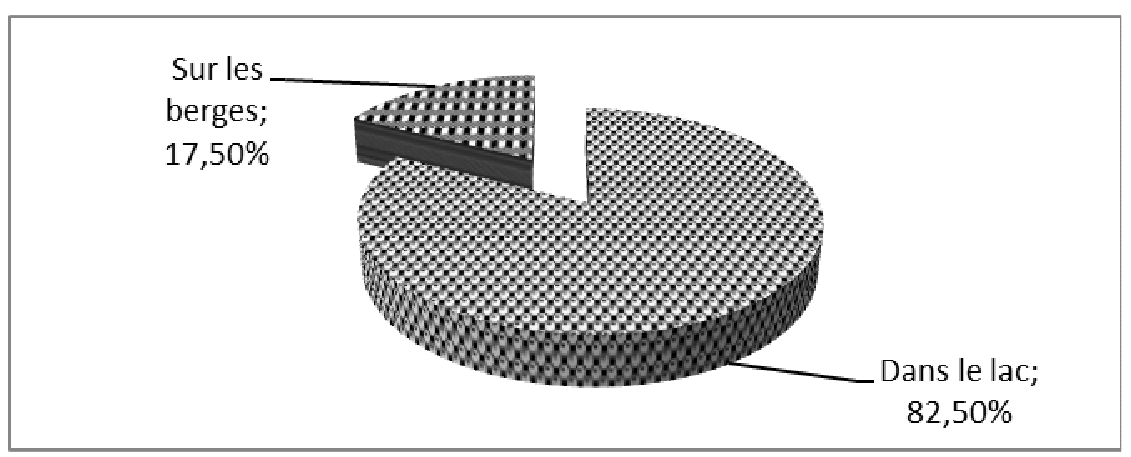

Figure 4 : Gestion des déchets liquides (eaux usées) par les enquêtés.

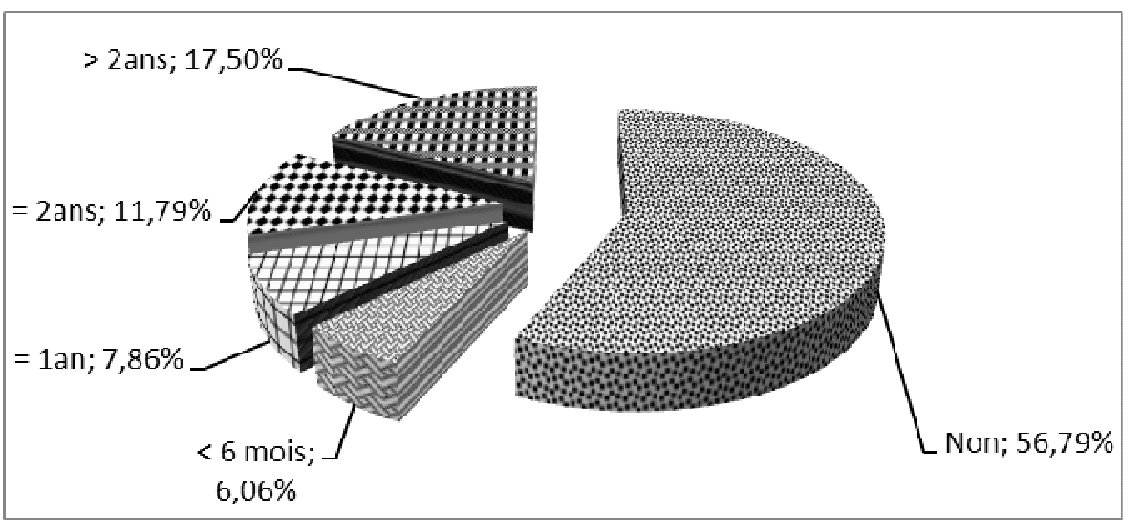

Figure 5 : Répartition des personnes enquêtées en fonction de leur dernière formation.

\section{DISCUSSION}

La population enquêtée est en légère majorité féminine comme le souligne les résultats du troisième Recensement Général de la Population et de l'Habitat (RGPH3) de
2002 qui révèlent que $51,46 \%$ de la population béninoise est féminine (INSAE, 2004). Cette population est aussi caractérisée par une forte dominance de jeunes. Cette observation est faite par Sèdogbo (2004) sur le 
lac Nokoué. Cette même observation est également faite dans la commune de Sô-Ava (Djènontin et Guidibi, 2006) et dans la commune de Sèmè-Podji (Akiyo et al., 2013).

Les résultats obtenus montrent également que ces populations du Lac Nokoué sont de plus en plus confrontées au problème d'hygiène et d'assainissement dû à l'absence de toilettes, de poubelles et de services d'assainissement adaptés. Cette situation peut être à l'origine d'une dégradation très poussée de l'environnement. Cela peut aussi entraîner plusieurs pathologies dans le milieu avec pour conséquences l'élévation du taux de morbidité. Cette observation est aussi faite par Sèdogbo (2004) sur le lac Nokoué, par Akiyo (2012) dans la commune de Sô-Âva et par Akiyo et al. (2013) dans la commune de Sèmè-Podji.

Les résultats de l'enquête montrent aussi que ces populations lacustres polluent constamment le lac de par les déchets résultant de leurs différentes activités (les fientes des animaux, les anciens branchages des acadjas et les produits pétroliers déversés dans le lac lors de son trafic). Cette observation est également faite par Sèdogbo (2004) et Akiyo (2012) sur le lac Nokoué et Agbohessi (2012) sur le lac Nokoué et la lagune de Porto-Novo.

Ces différentes observations montrent donc que la majorité des personnes enquêtées n'applique pas encore ou pas totalement les bonnes pratiques d'hygiène à cause de l'absence de formation ou d'une formation lointaine sur les bonnes pratiques d'hygiène.

\section{Conclusion}

L'étude a permis d'évaluer les bonnes pratiques d'hygiène au niveau des populations vivant aux abords immédiats le lac Nokoué. Les résultats révèlent que la plupart des personnes enquêtées ne pratiquent pas les règles d'hygiène malgré la formation de certains parmi eux. Le lac est donc constamment pollué par les déchets solides (ordures ménagères, selles, fientes des animaux domestiques) et liquides (eaux usées, urine) qu'ils produisent. Ce qui peut affecter la qualité sanitaire du lac donc directement ou indirectement celle des produits halieutiques qu'on $y$ trouve. Cela peut aussi causer diverses maladies ou pathologies liées au manque d'hygiène.

Il revient donc aux pouvoirs publics béninois de faire la promotion de l'hygiène en changeant et en améliorant les comportements et pratiques des populations lacustres en matière d'hygiène. Pour cela, il faut :

- organiser des campagnes d'informations, d'éducation et de sensibilisation des populations aux bonnes attitudes et pratiques d'hygiène pour une meilleure gestion du lac ;

- renforcer les capacités des responsables locaux ;

- assainir le lac en construisant plus de latrines et de douches publiques dans ces localités lacustres ;

- mettre en œuvre dans ces localités lacustres un dispositif de traitements des eaux usées et des déchets...

Ces différentes actions vont améliorer de façon quantitative et qualitative les conditions de vie de ces populations.

\section{CONFLITS D'INTERETS}

Les auteurs déclarent qu'ils n'ont aucun conflit d'intérêts pour cet article.

\section{CONTRIBUTIONS DES AUTEURS}

SPAS a élaboré les outils de collecte des données, a effectué l'enquête dans les différents villages ciblés et a participé à la rédaction de l'article. IBY a supervisé l'enquête et la rédaction de l'article. CCKT a participé à la réalisation des analyses statistiques des données. SBJB a participé à la réalisation des analyses statistiques des 
données. $\mathrm{RB}$ a participé à la réalisation de l'enquête. AAD a fait le dépouillement. GAM a supervisé la réalisation des analyses statistiques des données.

\section{REFERENCES}

Adjahouinou DC, Fiogbé ED. 2014. Concentrations of heavy metals in raw wastewaters of Cotonou city (Benin). $J$. Bio. \& Env. Sci., 5(2) : 534-541. DOI : http://innspub.net/wpcontent/uploads/2014/08/JBES-Vol5No2p534-541.pdf

Agbohessi TP, Toko II, Kestemont P. 2012. Etat des lieux de la contamination des écosystèmes aquatiques par les pesticides organochlorés dans le bassin cotonnier béninois. Cah. Agric., 21(1) : 46-56. DOI: 10.1684/agr.2012.0535

Agonkpahoun E. 2006. Evaluation de la pollution des eaux continentales par les métaux toxiques : Cas de la rivière Okpara et du lac Nokoué au Bénin. Thèse de Doctorat, Université d'AbomeyCalavi, Bénin, p. 87.

Akiyo OLR, Afouda AS, Yabi I, Adounkpé J, N'BESSA B. 2013. Impacts socioéconomiques et environnementaux de la promotion des ouvrages ECOSAN dans le développement de la commune de Sèmè-Podji au sud du Bénin. Int. J. Biol. Chem. Sci., 7(6): 2214-2232. DOI : http://dx.doi.org/10.4314/ijbcs.v7i6.4

Akiyo OLR. 2012. L'occupation de la Commune fluvio-lacustre de Sô-Ava : les fondements d'une adaptation originale et d'une mise en valeur d'un environnement spécifique du sud-Bénin. Thèse de Doctorat, Université d'Abomey-Calavi, Bénin, p. 372.

Dagnelie P. 1998. Théorie et méthodes statistiques (1ére Edition). Presse agronomique de Gembloux : Belgique.
Djénontin I, Guidibi EM. 2006. Monographie de la commune de SÔ-AVA (Bénin). Afrique Conseil : Bénin.

Dovonou F, Aïna M, Boukari M, Allassane A. 2011. Pollution physico-chimique et bactériologique d'un écosystème aquatique et ses risques écotoxicologiques : cas du lac Nokoué au Sud Bénin. Int. J. Biol. Chem. Sci., 5(4): 1590-1602.

DOI : http://indexmedicus.afro.who.int

Egounlety A. 2005. Etude de l'écologie et de l'exploitation des crevettes Peneidés du complexe lagunaire Lac Nokoué-Lagune Porto-Novo du Sud-Bénin. Mémoire, Université d'Abomey-Calavi, Bénin, p. 73.

Gnohossou MP. 2006. La faune benthique d'une lagune ouest africaine (le lac Nokoué au Bénin), diversité, abondance, variations Temporelles et spatiales, place dans la chaine trophique. Thèse de Doctorat, Institut National Polytechnique de Toulouse, France, p. 184.

INSAE (Institut National de la Statistique et de l'analyse économique). 2004. Cahier des villages et quartiers de ville du département de l'Atlantique (1ere Edition). Direction des Etudes Démographiques : Bénin.

Mama D. 2010. Méthodologie et résultats du diagnostic de l'eutrophisation du lac Nokoué (Bénin). Thèse de Doctorat, Université de Limoges, France, p. 177.

Mègnon GT, Soumanou MM, Tossou S, Mensah GA. 2012. Evaluation de la qualité sanitaire des crevettes (Penaeus sp) du Lac Nokoué au Sud-Bénin : Aspects chimique et microbiologique. Bulletin de la Recherche Agronomique du Bénin, Numéro spécial Elevages de gibier \& non gibier: 24-30. DOI : http://www.slire.net/download/1751/articl 
e_3_brab_n_sp_cial_gibier_non_gibier_ megnon

Pombo L, Elliot M, Rebelo JE. 2002. Changes in the fish fauna of the Rio de Aveiro estuarine lagoon (Portugal) during the twentieth century. Journal of Fish Biology, 61(sA) : 167 - 181. DOI : 10.1111/j.1095-8649.2002.tb01769.x

Rurangwa $E$, van den Berg $J$, Lalèyè $P A$, van Duijn AP, Rothuis A. 2014. Mission exploratoire Pêche, Pisciculture et Aquaculture au Bénin : Un quick scan du secteur pour des possibilités d'interventions (1ere Edition). Institute for Marine Resources \& Ecosystem Studies report: Pays-Bas.

Sachi SPA. 2015. Carte du lac Nokoué. Bénin, Octobre 2015.

Sèdogbo YA. 2004. Impact de la pollution environnementale sur la qualité des crevettes capturées au Bénin : cas des pêcheries du sud du lac Nokoué. Mémoire, Université d'Abomey-Calavi, Bénin, p. 67. 